\title{
ROBUST VARIABLE SELECTION BASED ON SCHWARZ INFORMATION CRITERION FOR LINEAR REGRESSION MODELS
}

\author{
SHOKRYA SALEH \\ Department of Mathematics, Jazan University, Saudia Arabia, Jazan \\ E-mails: salshekak@jazanu.edu.sa.
}

\begin{abstract}
Schwarz information criterion $(S I C)$ is a popular tool to select the best variables in regression data sets. However, $S I C$ defined using an unbounded estimator (Least Squares $(L S))$ which is very sensitive to the presence of outlying observations, especially bad leverage points. Thus, robust variable selection based on SIC for linear regression models is in need. This paper study the robust properties of $S I C$ derives its influence function and proposes robust $S I C$ based on the $M M$-estimation scale, aim to produce criterion which is effective in selecting accurate models in the presence of vertical outliers and high leverage points. The advantages of the proposed robust $S I C$ is demonstrated through simulation study and analysis of a real data set.
\end{abstract}

Keywords: Robust variable selection, robust regression, Influence function, Schwarz information criterion.

\section{Introduction}

This paper considers the problem of robust and selection variables for linear regression models. In modern regression data sets, outliers are commonly encountered in applications, which may appear either in response variables (vertical outliers) or in the predictors (leverage points). In this type of data set, it is difficult to select the best variables using criteria based on the classical estimator $(L S)$. Traditional selection criteria have a bad behavior with regards to robustness when vertical outliers in the data sets (see [1] and [2]). Moreover, they cannot be selected appropriate models for data with leverage points. Thus, robust variable selection methods for regression data are in need. Robust variable selection is one of the important topics in regression modeling; it gains the interest of many authors. For an instant, robust Mallow's $C p(R C p)$ proposed by [5], robust Akaike information criterion $(R A I C)$ proposed [6] and robust $R$-squared proposed by [7].

The Bayesian information criterion $(B I C)$ proposed by [3] is one of the commonly used 
criteria in model selection in linear regression. For a more general situation, [4] uses a Bayesian approach with a penalty term of the form $(p \log (n)) / n$, where $n$ is the sample size, and $p$ is the model dimension. Consider a linear regression model of the form

$$
y_{i}=\mu+\mathbf{X}_{i}^{T} \boldsymbol{\beta}+\epsilon_{i},
$$

where $\mu$ is the intercept parameter, $\mathbf{X}_{i}=\left(\mathbf{x}_{i 1}, \ldots, \mathbf{x}_{i p}\right)^{T}$ is a vector contains $p$ explanatory variables, $y_{i}$ is the response variable, $\boldsymbol{\beta}$ is a vector of $p$ parameters and $\epsilon_{i}$ is the error component that is independent and identically distributed (iid), with mean 0 and variance $\sigma^{2}$. The classical $S I C$ based on $L S$ estimate is defined as

$$
S I C_{L S}=\log \left(S S E_{p} / n\right)+(p \log (n)) / n,
$$

where $S S E_{p}=\sum_{i=1}^{n} r_{i}^{2}$, is the sum of squares error for sub model with $p$ variables and the residual $r_{i}=y_{i}-\hat{\mu}_{L S}-\mathbf{X}_{i}^{T} \hat{\boldsymbol{\beta}}_{L S}$. Therefore, models with values of $S I C_{L S}$ small will be preferred. Since the $L S$ estimator is vulnerable in the presence of outliers, it is not surprising that $S I C_{L S}$ inherits this problem. However, a robust version of $S I C$ based on $M$-estimators ([8]) proposed by [9], in this method replaced the squared residuals with a robust function $\rho$ and subsequently derived, $S I C_{M}=\sum_{i=1}^{n} \rho\left(r_{i} / \sigma\right)+(p \log (n)) / n$, where $\rho$ is a known function. Unfortunately, this criterion is not robust concerning contaminations in the predictor variables.

[10] proposed a robust version of SIC based on Least Trimmed Squares estimator (LTS) ([11]), named $S I C_{L T S}$ criterion. In a simulation study, [10] show that the $S I C_{L T S}$ can be robust for contamination in both the response and predictor variables. [10] discussed the influence of outliers on $S I C$ criterion, but the $L T S$ is highly inefficiency estimator when all the observations satisfy the regression model with normal errors.

[12] purpose $M M$-estimator of regression which having simultaneously, high breakdown point and high efficiency under normal errors; this estimator robust in a variety of contamination scenarios. However, $M M$-estimation is a combination of high breakdown value estimation and efficient estimation. $M M$-estimator does not use in $S I C$ criterion for variable selection aim. The purpose of this paper is to present $S I C_{M M}$ criterion for a robust variable selection criterion based on $M M$-scale estimates. The robust $S I C_{M M}$ allows to choose the best models, which fit the majority of the data by taking into account the presence of outliers and possible departures from the normality assumption on the error distribution.

The paper is organized as follows: Section 2 reviews the definition and some of the most important properties of $M M$-estimator in regression models. Section 3 define $S I C_{M M}$ criterion, study their robust properties, and describe an algorithm to compute $S I C_{M M}$ criterion. A simulation is conducted to study the performance of the proposed robust criterion in Section 4. Section 5 applies the robust criterion to the real data set. Finally, the concluding remark is present in Section 6 . 


\section{$2 \quad M M$-estimates}

$M M$-estimators proposed by [12] has become increasingly popular and one of the most commonly employed robust regression techniques. The $M M$-estimators reach a high level of robustness as well as high efficiency, by combining the properties of $M$-estimators ([8]) and $S$-estimators ([13]). The $M M$-estimators defined in three stages as follows:

Stage 1: take an initial estimate $\hat{\boldsymbol{\beta}}_{0}$ of $\hat{\boldsymbol{\beta}}$ in Equation (1) with a high breakdown point, possibly 0.5. The LTS estimation can be selected of $\hat{\boldsymbol{\beta}}_{0}$.

Stage 2: compute the residuals, $r_{i}=y_{i}-\hat{\mu}_{0}-\hat{\boldsymbol{\beta}}_{0} \mathbf{X}_{i}^{T}$ and compute the $M$-scale $\sigma\left(r_{i}\left(\hat{\boldsymbol{\beta}}_{0}\right)\right)$, defined as the value of $\sigma$ which is the solution of

$$
\frac{1}{n} \sum_{i=1}^{n} \rho_{0}\left(\left(r_{i}\left(\hat{\boldsymbol{\beta}}_{0}\right)\right) / \sigma\right)=b,
$$

where $b$ is constant defined by $E_{\Phi}\left(\rho\left(r_{i}\left(\hat{\boldsymbol{\beta}}_{0}\right)\right)=b\right.$, where $\Phi$ stands for the standard normal distribution. Using a function $\rho_{0}$ where satisfying following assumption $\left(A_{1}\right): \rho_{0}(0)=0$, $\rho_{0}(-u)=\rho_{0}(u)$, for $0 \leq u \leq v$ implies $\rho_{0}(u) \leq \rho_{0}(v), \rho_{0}$ is continuous , if $a=\sup \rho_{0}(u)$ , then $0 \leq a \leq \infty$, if $\rho_{0}(u)<a$ and $0 \leq u<v$, then $\rho_{0}(u) \leq \rho_{0}(v)$. Using a constant $b$ such that $b / a=0.5$, this implies that this scale estimate has breakdown point equal to 0.5 .

Stage 3: Let $\rho_{1}$ be another function satisfying: assumption (A1), $\rho_{1}(u) \leq \rho_{0}(u)$ and $\sup \rho_{1}(u)=\sup \rho_{0}(u)=a$. However, if $\psi_{1}=\rho_{1}^{\prime}$, then the $M M$-estimate $\left(\hat{\boldsymbol{\beta}}_{M M}\right)$ is defined as any solution of $\sum_{i=1}^{n} \psi_{1}\left(r_{i} / \sigma\right) \mathbf{X}_{i}=0, \quad \hat{\boldsymbol{\beta}}_{M M}$ obtained with iteratively reweighted least squares (IRWLS). [12] proved that $M M$-estimators are strongly consistent for $\hat{\boldsymbol{\beta}}_{0}$, besides, $M M$-estimator has simultaneously the two following properties:

1. Normal asymptotic efficiency.

2. Breakdown point greater than or equal to that of the initial estimator.

However, $M M$-estimator have the highest possible breakdown point equal to $50 \%$ (see $[14])$.

\section{$3 S I C_{M M}$ criterion for variable selection in linear re- gression}

This section discusses the possibility of extending the idea of using robust $M M$-estimators in the $S I C$. The $S I C$ method is expressed in terms of the variance, which are computed in $L S$ or robust method such as $M$ - or $L T S$ - estimation. [10] showed by derived the influence function of the $S I C$ criterion that, the robustness of the $S I C$ criterion will depend heavily on the robustness of the scale. In this study, instead of working with theses scales, a high breakdown point, and efficient $M M$-estimators for the $S I C$ criterion will use. This, in turn, reduces the effect of outliers and leverage points. Given scale 
Table 1: The simulated data set.

\begin{tabular}{cc}
\hline $\mathbf{X}_{i}$ & $y_{i}$ \\
\hline-1.2 & 1.2 \\
-1.15 & 1.35 \\
-1.1 & 1.02 \\
-1 & 0.95 \\
-0.95 & 1.05 \\
-0.9 & 0.73 \\
-0.85 & 0.91 \\
-0.8 & 0.85 \\
$\mathbf{x}_{10}$ & $y_{10}$ \\
0.8 & -0.88 \\
0.85 & -0.61 \\
0.9 & -0.81 \\
0.95 & -0.97 \\
1 & -1.18 \\
1.05 & -1.08 \\
1.1 & -0.99 \\
1.15 & -1.11 \\
1.2 & -1.14 \\
\hline
\end{tabular}

estimate of errors defined by $S=S S E_{p} /(n-p)$, with $r_{i}=y_{i}-\hat{\mu}_{M M}-\mathbf{X}_{i}^{T} \hat{\boldsymbol{\beta}}_{M M}$, then $S I C_{M M}$ criterion define as

$$
S I C_{M M}=\log \left(\frac{(n-p) S^{2}}{n}\right)+\frac{p \log (n)}{n} .
$$

The small value of $S I C_{M M}$ reveals that the explanatory variables adequately explain the distribution of $y$. Following same as experiment in [10], a set of independent random uniform variable $\mathbf{X}$ on $[-2,2]$ was generated according to the simple regression model, $y_{i}=\mathbf{X}_{i}+\epsilon_{i}, i=1, \ldots, 19$, where, $\epsilon_{i}$ are iid, normally distributed with expectation 0 and variance $\left(0.1^{2}\right)$, the data has been presented in Table 1 . The purpose of using this experiment to show the influence of an outlier on $S I C_{M M}$, this is illustrated through the presence of outliers in the $Y$-direction (vertical outlier) or in the $X$-direction (leverage point). For this, a point with coordinates $\left(0, y_{10}\right)$ is added, where the values of $y$ range between $(-1.5,3)$. A similar approach is performed for leverage points, that is, replacing the value $x$ with $\left(0, x_{10}\right)$, Figure 1 shows the situations of $y_{10}$ and $x_{10}$.

Figure 2 shows the results where the $S I C_{M M}$ shows a very robust behavior; there is only a slight loss in criteria, becoming constant when the outlier moves further away from the origin. Based on these results, it is evident that $S I C_{M M}$ show robust behavior in the presence of verticals or leverage point. In Section 4 simulation study and real data set illustrations clearly behavior of the proposed $S I C_{M M}$.

\subsection{Properties of the proposed robust $S I C_{M M}$ criterion}

\subsubsection{Influence function}

Consider the linear regression model in Equation ( 1) and assume that the distribution of errors are satisfying $F_{\sigma}(\mathbf{X})=F_{0}(\mathbf{X} / \sigma)$, where $\sigma$ is the residual scale parameter, and $F_{0}$ is symmetric, with a strictly positive density function.

Let $\mathbf{X}$ and $y$ be independent stochastic variables with distribution $H$. The functional $T$ is Fisher-consistent for the parameters $(\mu, \boldsymbol{\beta})$ at the model distribution $H$, which is as 

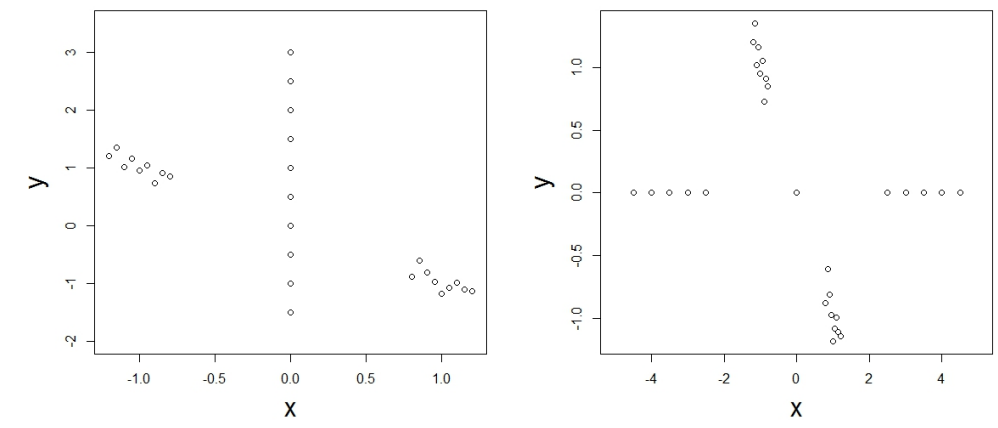

Figure 1: Data and positions for $y_{10}$ (left) and $x_{10}$ (right).
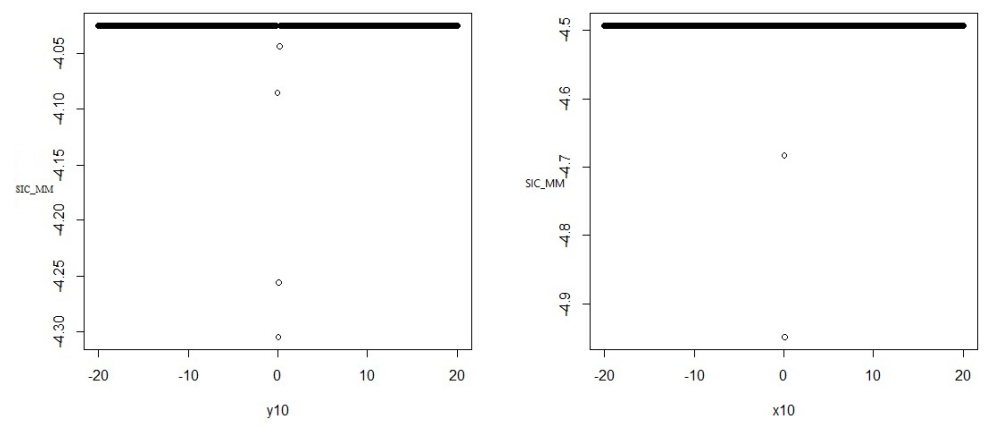

Figure 2: Effect of adding one observation $y_{10}$ (left) and $x_{10}$ (right) to the values of $S I C_{M M}$.

follows:

$$
T(H)=\left[\begin{array}{c}
a(H) \\
\mathbf{b}(H)
\end{array}\right]=\left[\begin{array}{l}
\mu \\
\boldsymbol{\beta}
\end{array}\right] .
$$

For a Fisher-consistent scale estimator, $T\left(F_{\sigma}\right)=\sigma$, for all $\sigma>0$. [14] defined the influence function of $T$ at the distribution $F$ as,

$$
I F((\mathbf{X}, y), T, H)=\lim _{\epsilon \rightarrow 0} \frac{T\left((1-\epsilon) H+\epsilon \Delta_{(\mathbf{X}, y)}\right)-T(H)}{\epsilon}=\frac{\partial}{\partial \epsilon}\left(T\left(\Delta_{(\mathbf{X}, y)}\right)\right) .
$$

where $T(H)$ is the function defined in the solution of the objective model and $\Delta_{(\mathbf{X}, y)}$ is the distribution contains outliers. The influence function measures the effect of possible outliers in the $S I C_{M M}$ criterion. It gives the amount of change in the model selection criterion estimator, caused by an infinitesimal amount of contamination at $(\mathbf{X}, y)$. Theorem 1 in [10] derived the influence function of $S I C$ based on scale estimates $S$ as follows:

$$
I F\left((\mathbf{X}, y), S I C_{S}, H\right)=2 n /(n-p) I F\left(r_{i} / \sigma_{S}, \hat{\sigma}_{S}^{2}, F_{0}\right),
$$

which is bounded in both $Y$ and $X$ directions, as $I F\left(r_{i} / \sigma, \hat{\sigma}_{S}^{2}, F_{0}\right)$ is bounded. Follows immediately from (6), the influence function of $S I C_{M M}$ is

$$
I F\left((\mathbf{X}, y), S I C_{M M}, H\right)=2 n /(n-p) \psi_{1}\left(r_{i}\right) \mathbf{X}_{i} \sigma_{0}^{2}\left(B\left(\psi_{1}, F_{0}\right) V\right)^{-1},
$$


where $V=E_{G_{0}}\left(\mathbf{X}_{i} \mathbf{X}_{i}^{T}\right)$ with $G_{0}$ has second moment, $B\left(\psi_{1}, F_{0}\right)=E_{F}\left(\psi_{1}\left(\frac{r_{i}}{\sigma_{0}}\right)\right)$ and $F$ is the distribution of the error $r_{i}$. Whereas, the influence function for the proposed criterion is bounded and note that a large zone of vertical outliers have zero influence, even when they are bad leverage points.

\subsubsection{The gross-error sensitivity of $S I C_{M M}$ criterion:}

[15] defined the gross-error sensitivity of an estimator $T$ at a distribution $F$ by

$$
\gamma^{\star}=\sup _{\mathbf{X}}|I F(\mathbf{X} ; T, F)|
$$

By taking the supreme over all $\mathbf{X}$ for which the $I F(\mathbf{X} ; T, F)$ exists, gross-error sensitivity measures the worst possible influence on an estimator by an arbitrary infinitesimal contaminant. If the gross-error sensitivity is unbounded, $\gamma^{\star}=\infty$, then the estimator is completely intolerant of outliers; a single outlier can ruin the estimator.

According to this definition, the gross-error sensitivity of the $S I C_{M M}$ criterion is defined as the supreme influence that observation can have. If $\hat{\boldsymbol{\beta}}_{M M}=0$, then $I F=0$, so it is assumed that $\hat{\boldsymbol{\beta}}_{M M} \neq 0$ then, if $\mathbf{X}$ tend to $\infty$, the gross-error sensitivity of will turn into:

$$
\gamma^{\star}\left(S I C_{M M}, F\right)=\sup (\mathbf{X}, y) I F\left((\mathbf{X}, y), S I C_{M M}, H\right)=2 n(n-p) E_{F_{0}}\left[\rho_{1}(\epsilon) \epsilon\right] \cdot \rho_{1}(\infty) .
$$

Briefly, if $\mathbf{X}$ tends to infinity, both $L S$ and $M$-estimators gain $\rho$ function yields high grosserror sensitivity. On the other hand, $M M$-estimator compute with $\rho$ function which yields the lowest $\gamma^{\star}$.

\section{Simulation study}

\subsection{Settings}

A simulation study was carried out to investigate the performance of the proposed robust $S I C_{M M}$ criterion. Furthermore, to compare this criterion with existing robust criteria, $S I C_{L T S}$ and $S I C_{M}$, and classical $S I C_{L S}$ criterion. For simplicity, considering the case when $p=3$, hence, the following set of parameters have to be estimated: $\left(\mu, \beta_{1}, \beta_{2}, \beta_{3}\right)$ and the set of different correlated random errors $\epsilon_{i}$ from the independent Normal distribution with mean 0 and variances $\sigma^{2}=0.7$.

The regression variables $\mathbf{x}_{i 1}, \mathbf{x}_{i 2}$ and $\mathbf{x}_{i 3}$ are generated in two different cases:

Case 1: independent uniform random variables on $[-1,1]$.

Case 2: correlated multivariate normal distribution, $N\left(0, \Sigma_{r}\right)$, for some $r \geq 0$, the variance matrix of the variables is defined by $\Sigma_{r, i, j}=r^{|i-j|}$ for $1 \leq i, j \leq 3, r=0.03,0.1,0.5$. Then the true model is given by: $y_{i}=\mu+\mathbf{x}_{i 1}+\mathbf{x}_{i 2}+\epsilon_{i}$. We then introduce vertical and leverage outliers into the data such that the percentages of contamination used are $\mathrm{c} \%=10 \%, 20 \%, 30 \%$ and $40 \%$ from two different sample sizes, namely $n=50$ and 100 - To investigate the robustness of the criteria against vertical and leverage outliers, the following scenarios were considered: 
(a) no contamination,

(b) vertical outliers (outliers in some $y_{i}$ only),

(c) good leverage points (outliers in the $y_{i}$ and $\mathbf{X}$ ),

(d) bad leverage points (outliers in some $\mathbf{X}$ only).

For vertical outliers, randomly generated different percentage of outliers from $N\left(50,0.1^{2}\right)$ for each of the simulated cases. For a good leverage point, considered the different percentages of outliers on the variables $\mathbf{x}_{1}$ and $\mathbf{x}_{2}$ are generated from $N\left(100,0.5^{2}\right)$ distribution, then generated $y$. For bad leverage points, different percentages of outliers on the variables $\mathbf{x}_{1}$ and $\mathbf{x}_{2}$ have generated from $N\left(100,0.5^{2}\right)$ distribution.

The performance of the criteria was then determined by assessing summary of the percentage over a simulation of selected following models : (i) correct fit (true model); (ii) over fit (models containing all the variables in the true model plus other variables that are redundant $\mathbf{x}_{1}, \mathbf{x}_{2}$, and $\mathbf{x}_{3}$ ); (iii) under fit (models with only a strict of the variables in true model); (iv) wrong fit (the model that are neither of the above). The simulations were performed by the statistical software $\mathrm{R}$ based on $\mathrm{s}=1000$ Monte Carlo trials, the function $r l m$ and ltsreg from the library (robust) was used for $M$ - and LTS-estimation, respectively, and function $l m r o b$ from library (robustbase) used for $M M$-estimation.

\subsection{Results and discussion}

First, consider the data without outliers, Table(2) shows detailed simulation results for two cases of simulation setting with all different $S I C$ criterion. The proposed $S I C_{M M}$ selects nearly $70 \%$ to $80 \%$ proportion of correct fit models, while the classical $S I C_{L S}$ performed better compared to robust $S I C$ with a high percentage (94\% t0 96\%), However, as the percentage of outliers increased (see, Table (3)), SIC $L S$ selected a larger proportion of wrong fit models than other criteria, this holds for both cases 1 and 2 . While the $S I C_{M}$ continues to yield a higher percentage of correct fit and these results hold as the percentage of vertical outliers increased to $20 \%$, then it tends to under fit. Thus, $S I C_{M}$ method ignored some of the important variables in the model. A higher proportion of over fit and correct fit models are select by $S I C_{L T S}$. As expected, the percentage of the true model in all cases of $S I C_{M M}$ was always large in the presence of vertical outliers, this result holds for both cases and with a high contamination level of vertical outliers. Table (4) Shows the situation where the data was contaminated with good leverage points, the results it can be concluded that good leverage points do not have much effect on all different SIC criteria. The presence of bad leverage points changes the picture dramatically. It can be observed from Tables (5) $S I C_{L S}$ and $S I C_{M}$ select a higher proportion of wrong fit than the $S I C$ based on $L T S$-estimators, $S I C_{L T S}$ tended to produce either correct fit or over fit model and the proposed criterion performed better when the bad leverage points are presents in the data.

In general, robust $S I C$ criteria with $M$ - and $L T S$-estimation are robust in the presence of outliers in the response variable. However, in the presence of bad leverage point, the value of these criteria will be affected and differs significantly from the true fit as the percentage of bad leverage point increases. But, $S I C_{M M}$ criterion less affected in all cases in the presence of outliers in $X$ and $Y$-directions. 
Table 2: Percentage of selected models from different criteria for data with no contamination.

\begin{tabular}{|c|c|c|c|c|c|c|c|c|c|c|c|}
\hline & & $n$ & $S I C_{M M}$ & $S I C_{L T S}$ & $S I C_{M}$ & $S I C_{L S}$ & $n$ & $S I C_{M M}$ & $S I C_{L T S}$ & $S I C_{M}$ & $S I C_{L S}$ \\
\hline \multirow[t]{4}{*}{ Case 1} & Correct fit & 50 & 70.6 & 36.3 & 55.6 & 94.8 & 100 & 79.3 & 48.3 & 64.1 & 96.6 \\
\hline & Under fit & & 4.2 & 2.8 & 10.0 & 0.1 & & 0.2 & 0.1 & 2.7 & 0.0 \\
\hline & Over fit & & 23.6 & 57.1 & 27.1 & 5.1 & & 20.5 & 50.3 & 31.0 & 3.4 \\
\hline & Wrong fit & & 1.6 & 3.8 & 7.3 & 0.0 & & 0.0 & 1.3 & 2.2 & 0.0 \\
\hline \multirow[t]{4}{*}{ Case 2, r $=0.03$} & Correct fit & 50 & 75.2 & 39.8 & 63.8 & 94 & 100 & 78.7 & 46.1 & 66.5 & 97.1 \\
\hline & Under fit & & 0.0 & 0.1 & 0.4 & 0 & & 0.0 & 0.0 & 0.0 & 0.0 \\
\hline & Over fit & & 24.6 & 59.8 & 35.1 & 6 & & 21.3 & 53.9 & 33.5 & 2.9 \\
\hline & Wrong fit & & 0.2 & 0.3 & 0.7 & 0 & & 0.0 & 0.0 & 0.0 & 0.0 \\
\hline \multirow[t]{4}{*}{ Case 2, r $=0.1$} & Correct fit & 50 & 75.8 & 36.9 & 64.0 & 93.6 & 100 & 78.9 & 49.1 & 65.8 & 97.7 \\
\hline & Under fit & & 0.2 & 0.4 & 0.9 & 0.0 & & 0.0 & 0.0 & 0.0 & 0.0 \\
\hline & Over fit & & 24.0 & 62.6 & 34.6 & 6.4 & & 21.1 & 50.9 & 34.2 & 2.3 \\
\hline & Wrong fit & & 0.0 & 0.1 & 0.5 & 0.0 & & 0.0 & 0.0 & 0.0 & 0.0 \\
\hline \multirow[t]{4}{*}{ Case 2, r $=0.5$} & Correct fit & 50 & 71.8 & 38.2 & 62.5 & 94.5 & 100 & 81.0 & 47.7 & 68.8 & 96.9 \\
\hline & Under fit & & 0.3 & 0.0 & 2.0 & 0.0 & & 0.1 & 0.2 & 0.2 & 0.0 \\
\hline & Over fit & & 27.4 & 60.8 & 33.5 & 5.5 & & 18.9 & 51.9 & 30.5 & 3.1 \\
\hline & Wrong fit & & 0.5 & 1.0 & 2.0 & 0.0 & & 0.0 & 0.2 & 0.5 & 0.0 \\
\hline
\end{tabular}

\section{$5 \quad$ Practical Example}

Hawkins-Bradu-Kass Data: This data has been generated by [16] for illustrating some of the merits of robust technique, the full data set is given in Table (6). They pointed out that the first 10 observations are bad leverage points; i.e. the first 10 observations are outliers and the next 4 observations are good leverage points. Figure (3) showed the regression plot of $y_{i}$ via different variables.

Table (7) shows the values of different criteria for Hawkins-Bradu-Kass data for different set of variables, where the small values of criteria are considered to show the best model. $S I C_{M M}$ agree on the importance of all three variables, which appears in a low value of $S I C_{M M}$. And the values of the other criteria are small with under fit values.
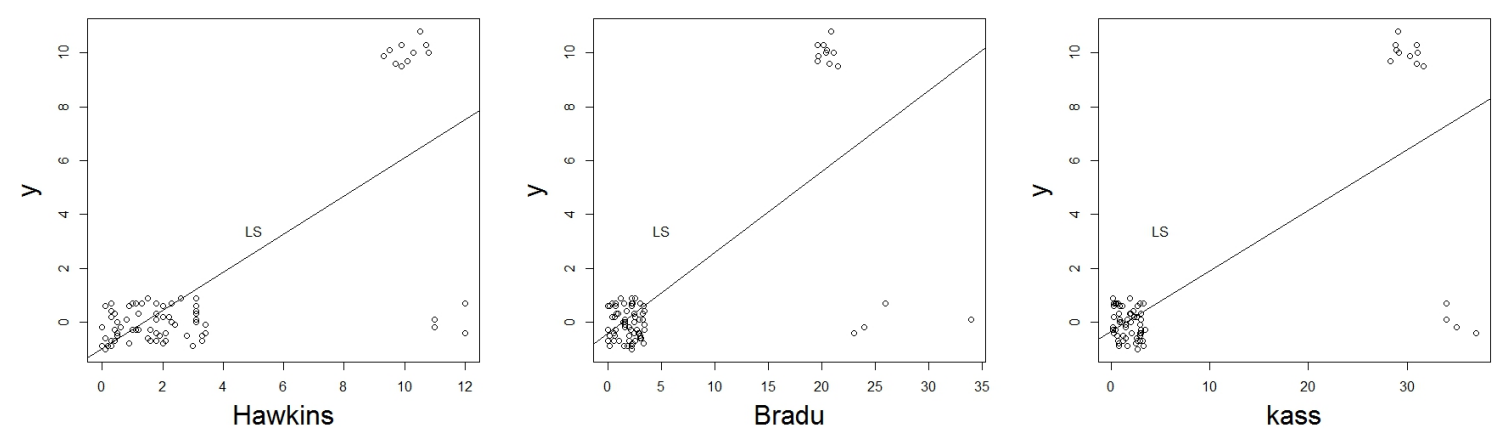

Figure 3: The regression plot of $y$ via Hawkins, Bradu, and Kass

\section{Conclusion}

In this article the $S I C$ criterion considered to be used with a high breakdown, efficient, and bounded influence scale estimators. The influence function of the criterion for the 
linear regression model based on the $M M$-scale approach was discussed. The simulation study and the application on real data set suggest that, at least for the scenarios considered, the proposed $S I C_{M M}$ criterion provide the best select the correct model for uncontaminated data sets, and stability in the presence of outliers.

\section{Acknowledgments}

This research has been funded by Jazan University, The author would like to thank Jazan University for their support to publish this study.

\section{References}

[1] Alshqaq, Shokrya Saleha A. "Robust Variable Selection in Linear Regression Models." PhD diss., Institut Sains Matematik, Fakulti Sains, Universiti Malaya, 2015.

[2] Saleh, Shokrya, and Ali Hassan Abuzaid. "Alternative Robust Variable Selection Procedures in Multiple Regression." Statistics, Optimization \& Information Computing 7, no. 4 (2019): 816-825.

[3] Akaike, Hirotugu. "A Bayesian analysis of the minimum AIC procedure." In Selected Papers of Hirotugu Akaike, pp. 275-280. Springer, New York, NY, 1998.

[4] Schwarz, G. "Estimating the dimension of a model The Annals of Statistics 6 (2), 461-464." URL: http://dx. doi. org/10.1214/aos/1176344136 (1978).

[5] Ronchetti, Elvezio, and Robert G. Staudte. "A robust version of Mallows's Cp." Journal of the American Statistical Association 89, no. 426 (1994): 550-559.

[6] Saleh, Shokrya. "Robust AIC with high breakdown scale estimate." Journal of Applied Mathematics 2014 (2014).

[7] Saleh, Shokrya. "Model selection via robust version of r-squared." Journal of Mathematics and Statistics 10, no. 3 (2014): 414-420.

[8] Huber, Peter J. "Robust regression: asymptotics, conjectures and Monte Carlo." The Annals of Statistics 1, no. 5 (1973): 799-821.

[9] Machado, Jose AF. "Robust model selection and M-estimation." Econometric Theory 9, no. 3 (1993): 478-493.

[10] Saleh, Shokrya, Nor Aishah Hamzah, and Rossita M. Yunus. "A robust version of Schwarz information criterion based on LTS." In AIP Conference Proceedings, vol. 1605, no. 1, pp. 967-972. American Institute of Physics, 2014.

[11] Rousseeuw, Peter J. "Least median of squares regression." Journal of the American statistical association 79, no. 388 (1984): 871-880. 
[12] Yohai, Victor J. "High breakdown-point and high efficiency robust estimates for regression." The Annals of Statistics (1987): 642-656.

[13] Rousseeuw, Peter, and Victor Yohai. "Robust regression by means of S-estimators." In Robust and nonlinear time series analysis, pp. 256-272. Springer, New York, NY, 1984.

[14] Hampel, Frank R., Elvezio M. Ronchetti, Peter J. Rousseeuw, and Werner A. Stahel. Robust statistics: the approach based on influence functions. Vol. 196. John Wiley \& Sons, 2011.

[15] Hampel, Frank R. "Contribution to the theory of robust estimation." Ph. D. Thesis, University of California, Berkeley (1968).

[16] Hawkins, Douglas M., Dan Bradu, and Gordon V. Kass. "Location of several outliers in multiple-regression data using elemental sets." Technometrics 26, no. 3 (1984): 197-208. 
Table 3: Percentage of selected models from different criteria for data with vertical outliers.

\begin{tabular}{|c|c|c|c|c|c|c|c|c|c|c|c|}
\hline $5 \%$ verticals & $n$ & & $S I C_{M M}$ & $S I C_{L T S}$ & $S I C_{M}$ & $S I C_{L S}$ & $n$ & $S I C_{M M}$ & $S I C_{L T S}$ & $S I C_{M}$ & $S I C_{L S}$ \\
\hline \multirow[t]{4}{*}{ Case 1} & 50 & Correct fit & 72.9 & 39.3 & 53.7 & 0.8 & 100 & 82.6 & 49.6 & 64.3 & 0.5 \\
\hline & & Under fit & 5.3 & 3.2 & 11.9 & 67.6 & & 0.2 & 0.4 & 3.1 & 69.3 \\
\hline & & Over fit & 19.9 & 54.4 & 27.1 & 0.1 & & 17.1 & 49.3 & 31.0 & 0.0 \\
\hline & & Wrong fit & 1.9 & 3.1 & 7.3 & 31.5 & & 0.1 & 0.7 & 1.6 & 30.2 \\
\hline Case 2, r=0.03 & 50 & Correct fit & 78.1 & 38.4 & 62.7 & 0.7 & 100 & 80.3 & 48.2 & 67.4 & 1.2 \\
\hline & & Under fit & 0.1 & 0.1 & 1.2 & 70.6 & & 0.0 & 0.0 & 0.0 & 77.7 \\
\hline & & Over fit & 21.8 & 61.2 & 35.5 & 0.2 & & 19.7 & 51.8 & 32.6 & 0.0 \\
\hline & & Wrong fit & 0.0 & 0.3 & 0.6 & 28.5 & & 0.0 & 0.0 & 0.0 & 21.1 \\
\hline Case 2, r $=0.1$ & 50 & Correct fit & 78.6 & 40.8 & 65.2 & 1.2 & 100 & 83 & 47.7 & 66.8 & 1.2 \\
\hline & & Under fit & 0.0 & 0.1 & 0.5 & 71.4 & & 0 & 0.0 & 0.0 & 77.5 \\
\hline & & Over fit & 21.4 & 59.1 & 34.0 & 0.3 & & 17 & 52.3 & 33.2 & 0.0 \\
\hline & & Wrong fit & 0.0 & 0.0 & 0.3 & 27.1 & & 0 & 0.0 & 0.0 & 21.3 \\
\hline Case 2, r $=0.5$ & 50 & Correct fit & 79.2 & 41.1 & 61.4 & 1.4 & 100 & 82.4 & 49.9 & 67.3 & 0.4 \\
\hline & & Under fit & 0.5 & 0.6 & 1.9 & 70.9 & & 0.0 & 0.0 & 0.0 & 78.8 \\
\hline & & Over fit & 19.8 & 57.6 & 33.8 & 0.1 & & 17.6 & 50.1 & 32.7 & 0.0 \\
\hline & & Wrong fit & 0.5 & 0.7 & 2.9 & 27.6 & & 0.0 & 0.0 & 0.0 & 20.8 \\
\hline $10 \%$ verticals & $n$ & & $S I C_{M M}$ & $S I C_{L T S}$ & $S I C_{M}$ & $S I C_{L S}$ & $n$ & $S I C_{M M}$ & $S I C_{L T S}$ & $S I C_{M}$ & $S I C_{L S}$ \\
\hline Case 1 & 50 & Correct fit & 77.8 & 40.7 & 57.3 & 0.8 & 100 & 82.2 & 51.6 & 65.6 & 0.3 \\
\hline & & Under fit & 3.2 & 3.4 & 9.1 & 66.6 & & 0.0 & 0.8 & 1.9 & 67.9 \\
\hline & & Over fit & 18.0 & 52.1 & 27.9 & 0.0 & & 17.7 & 47.1 & 30.7 & 0.0 \\
\hline & & Wrong fit & 1.0 & 3.8 & 5.7 & 32.6 & & 0.1 & 0.5 & 1.8 & 31.8 \\
\hline Case 2, r=0.03 & 50 & Correct fit & 83.4 & 44.3 & 64.6 & 0.8 & 100 & 84.5 & 52 & 66.5 & 0.8 \\
\hline & & Under fit & 0.0 & 0.1 & 0.6 & 69.0 & & 0.0 & 0 & 0.0 & 68.1 \\
\hline & & Over fit & 16.6 & 55.4 & 34.6 & 0.0 & & 15.5 & 48 & 33.5 & 0.0 \\
\hline & & Wrong fit & 0.0 & 0.2 & 0.2 & 30.2 & & 0.0 & 0 & 0.0 & 31.1 \\
\hline Case 2, r=0.1 & 50 & Correct fit & 80.7 & 41.4 & 64.8 & 0.9 & 100 & 85.3 & 55.1 & 67.2 & 0.3 \\
\hline & & Under fit & 0.2 & 0.3 & 0.9 & 68.3 & & 0.0 & 0.0 & 0.0 & 73.4 \\
\hline & & Over fit & 19.0 & 58.0 & 33.6 & 0.2 & & 14.7 & 44.9 & 32.8 & 0.1 \\
\hline & & Wrong fit & 0.1 & 0.3 & 0.7 & 30.6 & & 0.0 & 0.0 & 0.0 & 26.2 \\
\hline Case 2, r=0.5 & 50 & Correct fit & 80.0 & 42.4 & 62.7 & 0.7 & 100 & 85 & 51.2 & 68.7 & 0.7 \\
\hline & & Under fit & 0.1 & 0.4 & 1.9 & 67.0 & & 0 & 0.0 & 0.1 & 72.1 \\
\hline & & Over fit & 19.8 & 56.2 & 33.4 & 0.0 & & 15 & 48.7 & 31.1 & 0.0 \\
\hline & & Wrong fit & 0.1 & 1.0 & 2.0 & 32.3 & & 0 & 0.1 & 0.1 & 27.2 \\
\hline $20 \%$ verticals & $n$ & & $S I C_{M M}$ & $S I C_{L T S}$ & $S I C_{M}$ & $S I C_{L S}$ & $n$ & $S I C_{M M}$ & $S I C_{L T S}$ & $S I C_{M}$ & $S I C_{L S}$ \\
\hline Case 1 & 50 & Correct fit & 85.5 & 48.0 & 54.9 & 0.7 & 100 & 88.5 & 58.0 & 66.8 & 0.6 \\
\hline & & Under fit & 4.0 & 4.9 & 13.4 & 64.8 & & 0.0 & 0.1 & 3.5 & 69.0 \\
\hline & & Over fit & 9.9 & 43.6 & 22.8 & 0.0 & & 11.4 & 41.1 & 28.1 & 0.0 \\
\hline & & Wrong fit & 0.6 & 3.5 & 8.9 & 34.5 & & 0.1 & 0.8 & 1.6 & 30.4 \\
\hline Case 2, r=0.03 & 50 & Correct fit & 88.1 & 51.2 & 67.6 & 0.4 & 100 & 90.4 & 59.6 & 66.7 & 0.3 \\
\hline & & Under fit & 0.0 & 0.1 & 1.1 & 63.0 & & 0.0 & 0.0 & 0.0 & 69.6 \\
\hline & & Over fit & 11.8 & 48.5 & 30.2 & 0.1 & & 9.6 & 40.4 & 33.3 & 0.0 \\
\hline & & Wrong fit & 0.1 & 0.2 & 1.1 & 36.5 & & 0.0 & 0.0 & 0.0 & 30.1 \\
\hline Case 2, r=0.1 & 50 & Correct fit & 86.3 & 51.0 & 67.8 & 0.4 & 100 & 89.5 & 63 & 68.1 & 0.5 \\
\hline & & Under fit & 0.0 & 0.3 & 1.8 & 68.5 & & 0.0 & 0 & 0.0 & 69.2 \\
\hline & & Over fit & 13.6 & 48.6 & 29.4 & 0.1 & & 10.5 & 37 & 31.8 & 0.0 \\
\hline & & Wrong fit & 0.1 & 0.1 & 1.0 & 31.0 & & 0.0 & 0 & 0.1 & 30.3 \\
\hline Case 2, r=0.5 & 50 & Correct fit & 86.2 & 49.9 & 63.9 & 0.7 & 100 & 89.5 & 62.6 & 67.7 & 0.1 \\
\hline & & Under fit & 0.2 & 0.5 & 3.4 & 64.6 & & 0.0 & 0.0 & 0.3 & 69.4 \\
\hline & & Over fit & 13.3 & 48.6 & 29.4 & 0.1 & & 10.5 & 37.3 & 31.9 & 0.0 \\
\hline & & Wrong fit & 0.3 & 1.0 & 3.3 & 34.6 & & 0.0 & 0.1 & 0.1 & 30.5 \\
\hline $30 \%$ vertical & $n$ & & $S I C_{M M}$ & $S I C_{L T S}$ & $S I C_{M}$ & $S I C_{L S}$ & $n$ & $S I C_{M M}$ & $S I C_{L T S}$ & $S I C_{M}$ & $S I C_{L S}$ \\
\hline Case 1 & 50 & Correct fit & 89.2 & 57.0 & 4.7 & 0.2 & 100 & 94.1 & 69.7 & 8.3 & 0.3 \\
\hline & & Under fit & 3.0 & 4.4 & 60.0 & 66.0 & & 0.1 & 0.4 & 60.3 & 68.9 \\
\hline & & Over fit & 7.0 & 37.0 & 1.7 & 0.0 & & 5.8 & 29.8 & 0.7 & 0.0 \\
\hline & & Wrong fit & 0.8 & 1.6 & 33.6 & 33.8 & & 0.0 & 0.1 & 30.7 & 30.8 \\
\hline Case 2, r=0.03 & 50 & Correct fit & 92.7 & 61.2 & 7.7 & 0.2 & 100 & 93.7 & 71.7 & 15.6 & 0.4 \\
\hline & & Under fit & 0.0 & 0.1 & 63.3 & 67.0 & & 0.0 & 0.0 & 58.8 & 68.1 \\
\hline & & Over fit & 7.3 & 38.7 & 1.7 & 0.1 & & 6.3 & 28.3 & 2.2 & 0.0 \\
\hline & & Wrong fit & 0.0 & 0.0 & 27.3 & 32.7 & & 0.0 & 0.0 & 23.4 & 31.5 \\
\hline Case 2, r=0.1 & 50 & Correct fit & 93.1 & 59.8 & 8.3 & 0.6 & 100 & 94 & 69.8 & 18.2 & 0.0 \\
\hline & & Under fit & 0.0 & 0.1 & 60.2 & 66.4 & & 0 & 0.0 & 57.8 & 67.2 \\
\hline & & Over fit & 6.9 & 40.0 & 1.3 & 0.0 & & 6 & 30.2 & 1.0 & 0.0 \\
\hline & & Wrong fit & 0.0 & 0.1 & 30.2 & 33.0 & & 0 & 0.0 & 23.0 & 32.8 \\
\hline Case 2, r $=0.5$ & 50 & Correct fit & 91.6 & 58.3 & 7.5 & 0.4 & 100 & 94.4 & 73.1 & 15.4 & 0.2 \\
\hline & & Under fit & 0.3 & 0.3 & 65.5 & 63.5 & & 0.0 & 0.0 & 60.5 & 66.1 \\
\hline & & Over fit & 8.0 & 40.7 & 1.6 & 0.2 & & 5.6 & 26.9 & 1.4 & 0.1 \\
\hline & & Wrong fit & 0.1 & 0.7 & 25.4 & 35.9 & & 0.0 & 0.0 & 22.7 & 33.6 \\
\hline $40 \%$ verticals & $n$ & & $S I C_{M M}$ & $S I C_{L T S}$ & $S I C_{M}$ & $S I C_{L S}$ & $n$ & $S I C_{M M}$ & $S I C_{L T S}$ & $S I C_{M}$ & $S I C_{L S}$ \\
\hline Case 1 & 50 & Correct fit & 86.6 & 69.4 & 5.3 & 0.4 & 100 & 97.1 & 81.7 & 1.1 & 0.1 \\
\hline & & Under fit & 10.9 & 5.2 & 61.9 & 68.0 & & 0.1 & 0.2 & 69.1 & 67.5 \\
\hline & & Over fit & 1.8 & 24.4 & 3.6 & 0.0 & & 2.8 & 18.1 & 0.9 & 0.0 \\
\hline & & Wrong fit & 0.7 & 1.0 & 29.2 & 31.6 & & 0.0 & 0.0 & 28.9 & 32.4 \\
\hline Case 2, r=0.03 & 50 & Correct fit & 97.3 & 74.0 & 2.6 & 0.1 & 100 & 98.4 & 82.7 & 3.8 & 0.1 \\
\hline & & Under fit & 0.0 & 0.1 & 68.2 & 66.1 & & 0.0 & 0.0 & 77.6 & 67.4 \\
\hline & & Over fit & 2.7 & 25.9 & 2.9 & 0.0 & & 1.6 & 17.3 & 0.5 & 0.0 \\
\hline & & Wrong fit & 0.0 & 0.0 & 26.3 & 33.8 & & 0.0 & 0.0 & 18.1 & 32.5 \\
\hline Case 2, r=0.1 & 50 & Correct fit & 98.8 & 76.2 & 3.8 & 0.8 & 100 & 97.9 & 81.7 & 2.4 & 0.2 \\
\hline & & Under fit & 0.0 & 0.1 & 67.8 & 65.2 & & 0.0 & 0.0 & 77.0 & 67.3 \\
\hline & & Over fit & 1.2 & 23.7 & 2.4 & 0.0 & & 2.1 & 18.3 & 0.3 & 0.0 \\
\hline & & Wrong fit & 0.0 & 0.0 & 26.0 & 34.0 & & 0.0 & 0.0 & 20.3 & 32.5 \\
\hline Case 2, r=0.5 & 50 & Correct fit & 98.1 & 74.9 & 3.4 & 1.2 & 100 & 98.1 & 82.5 & 2.0 & 0.9 \\
\hline & & Under fit & 0.1 & 0.4 & 68.8 & 64.8 & & 0.0 & 0.0 & 78.7 & 67.6 \\
\hline & & Over fit & 1.8 & 24.5 & 3.6 & 0.3 & & 1.9 & 17.5 & 1.0 & 0.0 \\
\hline & & Wrong fit & 0.0 & 0.2 & 24.2 & 33.7 & & 0.0 & 0.0 & 18.3 & 31.5 \\
\hline
\end{tabular}


Table 4: Percentage of selected models from different criteria for data with good leverage points

\begin{tabular}{|c|c|c|c|c|c|c|c|c|c|c|c|}
\hline $5 \%$ good leverage & $n$ & & $S I C_{M M}$ & $S I C_{L T S}$ & $S I C_{M}$ & $S I C_{L S}$ & $n$ & $S I C_{M M}$ & $S I C_{L T S}$ & $S I C_{M}$ & $S I C_{L S}$ \\
\hline \multirow[t]{4}{*}{ Case 1} & 50 & Correct fit & 93.4 & 67.5 & 76.0 & 100 & 100 & 97.3 & 78.1 & 84.9 & 100 \\
\hline & & Under fit & 2.1 & 2.6 & 3.4 & 0 & & 0.2 & 0.2 & 0.2 & 0 \\
\hline & & Over fit & 3.9 & 26.9 & 19.0 & 0 & & 2.5 & 21.2 & 14.7 & 0 \\
\hline & & Wrong fit & 0.6 & 3.0 & 1.6 & 0 & & 0.0 & 0.5 & 0.2 & 0 \\
\hline Case 2, r $=0.03$ & 50 & Correct fit & 96.0 & 69.4 & 78.9 & 100 & 100 & 97.4 & 82.5 & 87.3 & 100 \\
\hline & & Under fit & 0.0 & 0.6 & 1.0 & 0 & & 0.0 & 0.0 & 0.1 & 0 \\
\hline & & Over fit & 3.8 & 29.1 & 19.7 & 0 & & 2.6 & 17.5 & 12.5 & 0 \\
\hline & & Wrong fit & 0.2 & 0.9 & 0.4 & 0 & & 0.0 & 0.0 & 0.1 & 0 \\
\hline Case 2, r=0.1 & 50 & Correct fit & 94.6 & 70.9 & 76.2 & 100 & 100 & 97.5 & 79.1 & 88.3 & 100 \\
\hline & & Under fit & 0.1 & 0.3 & 1.6 & 0 & & 0.0 & 0.0 & 0.0 & 0 \\
\hline & & Over fit & 4.8 & 27.6 & 21.0 & 0 & & 2.5 & 20.9 & 11.7 & 0 \\
\hline & & Wrong fit & 0.5 & 1.2 & 1.2 & 0 & & 0.0 & 0.0 & 0.0 & 0 \\
\hline Case 2, r $=0.5$ & 50 & Correct fit & 94.0 & 70.7 & 78.6 & 100 & 100 & 97.6 & 76.9 & 85.6 & 100 \\
\hline & & Under fit & 0.3 & 0.4 & 1.3 & 0 & & 0.0 & 0.0 & 0.0 & 0 \\
\hline & & Over fit & 5.3 & 28.1 & 19.6 & 0 & & 2.4 & 23.1 & 14.4 & 0 \\
\hline & & Wrong fit & 0.4 & 0.8 & 0.5 & 0 & & 0.0 & 0.0 & 0.0 & 0 \\
\hline $10 \%$ good leverage & $n$ & & $S I C_{M M}$ & $S I C_{L T S}$ & $S I C_{M}$ & $S I C_{L S}$ & $n$ & $S I C_{M M}$ & $S I C_{L T S}$ & $S I C_{M}$ & $S I C_{L S}$ \\
\hline Case 1 & 50 & Correct fit & 97.2 & 81.2 & 86.0 & 100 & 100 & 100 & 94.0 & 96.4 & 100 \\
\hline & & Under fit & 0.8 & 2.3 & 2.5 & 0 & & 0 & 0.4 & 0.6 & 0 \\
\hline & & Over fit & 1.5 & 14.1 & 9.4 & 0 & & 0 & 5.6 & 2.9 & 0 \\
\hline & & Wrong fit & 0.5 & 2.4 & 2.1 & 0 & & 0 & 0.0 & 0.1 & 0 \\
\hline Case 2, r $=0.03$ & 50 & Correct fit & 98.7 & 85.6 & 90.2 & 100 & 100 & 99.7 & 94.3 & 95.9 & 100 \\
\hline & & Under fit & 0.0 & 0.0 & 0.0 & 0 & & 0.0 & 0.0 & 0.0 & 0 \\
\hline & & Over fit & 1.3 & 14.4 & 9.8 & 0 & & 0.3 & 5.7 & 4.1 & 0 \\
\hline & & Wrong fit & 0.0 & 0.0 & 0.0 & 0 & & 0.0 & 0.0 & 0.0 & 0 \\
\hline Case 2, r=0.1 & 50 & Correct fit & 98.5 & 83.8 & 90.1 & 100 & 100 & 100 & 94.8 & 96.9 & 100 \\
\hline & & Under fit & 0.0 & 0.0 & 0.0 & 0 & & 0 & 0.0 & 0.0 & 0 \\
\hline & & Over fit & 1.5 & 16.1 & 9.8 & 0 & & 0 & 5.2 & 3.1 & 0 \\
\hline & & Wrong fit & 0.0 & 0.1 & 0.1 & 0 & & 0 & 0.0 & 0.0 & 0 \\
\hline Case 2, r $=0.5$ & 50 & Correct fit & 98.0 & 82.6 & 88.9 & 100 & 100 & 99.9 & 94 & 95.1 & 100 \\
\hline & & Under fit & 0.3 & 0.5 & 1.1 & 0 & & 0.0 & 0 & 0.1 & 0 \\
\hline & & Over fit & 1.5 & 15.7 & 9.1 & 0 & & 0.1 & 6 & 4.8 & 0 \\
\hline & & Wrong fit & 0.2 & 1.2 & 0.9 & 0 & & 0.0 & 0 & 0.0 & 0 \\
\hline $20 \%$ good leverage & $n$ & & $S I C_{M M}$ & $S I C_{L T S}$ & $S I C_{M}$ & $S I C_{L S}$ & $n$ & $S I C_{M M}$ & $S I C_{L T S}$ & $S I C_{M}$ & $S I C_{L S}$ \\
\hline Case 1 & 50 & Correct fit & 98.6 & 94.7 & 92.8 & 100 & 100 & 99.9 & 94 & 95.1 & 100 \\
\hline & & Under fit & 0.8 & 1.2 & 3.7 & 0 & & 0.0 & 0 & 0.1 & 0 \\
\hline & & Over fit & 0.1 & 1.1 & 0.6 & 0 & & 0.1 & 6 & 4.8 & 0 \\
\hline & & Wrong fit & 0.5 & 3.0 & 2.9 & 0 & & 0.0 & 0 & 0.0 & 0 \\
\hline Case 2, r=0.03 & 50 & Correct fit & 100 & 98.7 & 99.3 & 100 & 100 & 100 & 99.9 & 100 & 100 \\
\hline & & Under fit & 0 & 0.0 & 0.2 & 0 & & 0 & 0.0 & 0 & 0 \\
\hline & & Over fit & 0 & 1.2 & 0.5 & 0 & & 0 & 0.1 & 0 & 0 \\
\hline & & Wrong fit & 0 & 0.1 & 0.0 & 0 & & 0 & 0.0 & 0 & 0 \\
\hline Case 2, r=0.1 & 50 & Correct fit & 100 & 98.5 & 99.2 & 100 & 100 & 100 & 99.9 & 100 & 100 \\
\hline & & Under fit & 0 & 0.0 & 0.1 & 0 & & 0 & 0.0 & 0 & 0 \\
\hline & & Over fit & 0 & 1.4 & 0.4 & 0 & & 0 & 0.1 & 0 & 0 \\
\hline & & Wrong fit & 0 & 0.1 & 0.3 & 0 & & 0 & 0.0 & 0 & 0 \\
\hline Case 2, r=0.5 & 50 & Correct fit & 99.8 & 97.3 & 97.2 & 100 & 100 & 100 & 99.7 & 99.9 & 100 \\
\hline & & Under fit & 0.1 & 0.5 & 1.2 & 0 & & 0 & 0.0 & 0.1 & 0 \\
\hline & & Over fit & 0.0 & 1.1 & 0.2 & 0 & & 0 & 0.1 & 0.0 & 0 \\
\hline & & Wrong fit & 0.1 & 1.1 & 1.4 & 0 & & 0 & 0.2 & 0.0 & 0 \\
\hline $30 \%$ good leverage & $n$ & & $S I C_{M M}$ & $S I C_{L T S}$ & $S I C_{M}$ & $S I C_{L S}$ & $n$ & $S I C_{M M}$ & $S I C_{L T S}$ & $S I C_{M}$ & $S I C_{L S}$ \\
\hline Case 1 & 50 & Correct fit & 99.0 & 96.9 & 94.3 & 100 & 100 & 100 & 99.8 & 99.2 & 100 \\
\hline & & Under fit & 0.5 & 0.6 & 2.4 & 0 & & & 0.1 & 0.4 & 0 \\
\hline & & Over fit & 0.0 & 0.1 & 0.0 & 0 & & 0.0 & 0.0 & 0 & \\
\hline & & Wrong fit & 0.5 & 2.4 & 3.3 & 0 & & 0 & 0.1 & 0.4 & 0 \\
\hline Case 2, r=0.03 & 50 & Correct fit & 100 & 99.9 & 99.5 & 100 & 100 & 100 & 100 & 100 & 100 \\
\hline & & Under fit & 0 & 0.0 & 0.0 & 0 & & 0 & 0 & 0 & 0 \\
\hline & & Over fit & 0 & 0.0 & 0.0 & 0 & & 0 & 0 & 0 & 0 \\
\hline & & Wrong fit & 0 & 0.1 & 0.5 & 0 & & 0 & 0 & 0 & 0 \\
\hline Case 2, r=0.1 & 50 & Correct fit & 100 & 99.4 & 99.2 & 100 & 100 & 100 & 100 & 100 & 100 \\
\hline & & Under fit & 0 & 0.1 & 0.1 & 0 & & 0 & 0 & 0 & 0 \\
\hline & & Over fit & 0 & 0.1 & 0.0 & 0 & & 0 & 0 & 0 & 0 \\
\hline & & Wrong fit & 0 & 0.4 & 0.7 & 0 & & 0 & 0 & 0 & 0 \\
\hline Case 2, r=0.5 & 50 & Correct fit & 99.8 & 97.9 & 95.9 & 100 & 100 & 100 & 99.7 & 99.4 & 100 \\
\hline & & Under fit & 0.0 & 0.1 & 2.3 & 0 & & 0 & 0.1 & 0.3 & 0 \\
\hline & & Over fit & 0.0 & 0.0 & 0.0 & 0 & & 0 & 0.0 & 0.0 & 0 \\
\hline & & Wrong fit & 0.2 & 2.0 & 1.8 & 0 & & 0 & 0.2 & 0.3 & 0 \\
\hline $40 \%$ good leverage & $n$ & & $S I C_{M M}$ & $S I C_{L T S}$ & $S I C_{M}$ & $S I C_{L S}$ & $n$ & $S I C_{M M}$ & $S I C_{L T S}$ & $S I C_{M}$ & $S I C_{L S}$ \\
\hline Case 1 & 50 & Correct fit & 98.8 & 96.0 & 93.5 & 100 & 100 & 100 & 99.7 & 99.0 & 100 \\
\hline & & Under fit & 0.6 & 1.2 & 4.1 & 0 & & 0 & 0.0 & 0.7 & 0 \\
\hline & & Over fit & 0.0 & 0.0 & 0.0 & 0 & & 0 & 0.0 & 0.0 & 0 \\
\hline & & Wrong fit & 0.6 & 2.8 & 2.4 & 0 & & 0 & 0.3 & 0.3 & 0 \\
\hline Case 2, r $=0.03$ & 50 & Correct fit & 99.8 & 99.4 & 98 & 100 & 100 & 100 & 100 & 100 & 100 \\
\hline & & Under fit & 0.1 & 0.1 & 1 & 0 & & 0 & 0 & 0 & 0 \\
\hline & & Over fit & 0.0 & 0.0 & 0 & 0 & & 0 & 0 & 0 & 0 \\
\hline & & Wrong fit & 0.1 & 0.5 & 1 & 0 & & 0 & 0 & 0 & 0 \\
\hline Case 2, r $=0.1$ & 50 & Correct fit & 99.8 & 99.3 & 98.9 & 100 & 100 & 100 & 100 & 99.8 & 100 \\
\hline & & Under fit & 0.1 & 0.4 & 0.4 & 0 & & 0 & 0 & 0.1 & 0 \\
\hline & & Over fit & 0.0 & 0.0 & 0.0 & 0 & & 0 & 0 & 0.0 & 0 \\
\hline & & Wrong fit & 0.1 & 0.3 & 0.7 & 0 & & 0 & 0 & 0.1 & 0 \\
\hline Case 2, r=0.5 & 50 & Correct fit & 98.9 & 97.3 & 94.9 & 100 & 100 & 100 & 100 & 99.9 & 100 \\
\hline & & Under fit & 0.5 & 0.7 & 2.8 & 0 & & 0 & 0 & 0.1 & 0 \\
\hline & & Over fit & 0.0 & 0.0 & 0.0 & 0 & & 0 & 0 & 0.0 & 0 \\
\hline & & Wrong fit & 0.6 & 2.0 & 2.3 & 0 & & 0 & 0 & 0.0 & 0 \\
\hline
\end{tabular}


Table 5: Percentage of selected models from different criteria for data with bad leverage points

\begin{tabular}{|c|c|c|c|c|c|c|c|c|c|c|c|}
\hline $5 \%$ bad leverage & $n$ & & $S I C_{M M}$ & $S I C_{L T S}$ & $S I C_{M}$ & $S I C_{L S}$ & $n$ & $S I C_{M M}$ & $S I C_{L T S}$ & $S I C_{M}$ & $S I C_{L S}$ \\
\hline \multirow[t]{4}{*}{ Case 1} & Correct fit & 50 & 71.8 & 36.3 & 19.6 & 1.8 & 100 & 80.9 & 47.1 & 15.2 & 1.1 \\
\hline & Under fit & & 3.8 & 3.0 & 30.7 & 49.0 & & 0.0 & 0.8 & 35.2 & 49.2 \\
\hline & Over fit & & 21.2 & 55.9 & 9.3 & 0.0 & & 19.0 & 51.6 & 8.4 & 0.0 \\
\hline & Wrong fit & & 3.2 & 4.8 & 40.4 & 49.2 & & 0.1 & 0.5 & 41.2 & 49.7 \\
\hline Case 2, r $=0.03$ & Correct fit & 50 & 75.5 & 39.6 & 17.1 & 4.0 & 100 & 83.2 & 51.4 & 19.4 & 3.7 \\
\hline & Under fit & & 0.1 & 0.3 & 32.4 & 50.9 & & 0.0 & 0.0 & 31.7 & 48.3 \\
\hline & Over fit & & 24.4 & 60.1 & 8.7 & 0.1 & & 16.8 & 48.6 & 7.8 & 0.0 \\
\hline & Wrong fit & & 0.0 & 0.0 & 41.8 & 45.0 & & 0.0 & 0.0 & 41.1 & 48.0 \\
\hline Case 2, r $=0.1$ & Correct fit & 50 & 77.5 & 40.1 & 18.6 & 4.0 & 100 & 79.9 & 50.6 & 14.4 & 2.7 \\
\hline & Under fit & & 0.0 & 0.2 & 31.5 & 47.0 & & 0.0 & 0.0 & 35.4 & 45.0 \\
\hline & Over fit & & 22.5 & 59.7 & 9.9 & 0.1 & & 20.1 & 49.4 & 9.7 & 0.1 \\
\hline & Wrong fit & & 0.0 & 0.0 & 40.0 & 48.9 & & 0.0 & 0.0 & 40.5 & 52.2 \\
\hline Case 2, r $=0.5$ & Correct fit & 50 & 80.1 & 37.9 & 9.5 & 0.4 & 100 & 80.5 & 50.4 & 6.1 & 0.1 \\
\hline & Under fit & & 0.4 & 0.6 & 16.6 & 2.9 & & 0.0 & 0.0 & 12.2 & 0.4 \\
\hline & Over fit & & 19.5 & 60.9 & 19.4 & 4.1 & & 19.5 & 49.6 & 22.6 & 4.9 \\
\hline & Wrong fit & & 0.0 & 0.6 & 54.5 & 92.6 & & 0.0 & 0.0 & 59.1 & 94.6 \\
\hline $10 \%$ bad leverage & $n$ & & $S I C_{M M}$ & $S I C_{L T S}$ & $S I C_{M}$ & $S I C_{L S}$ & $n$ & $S I C_{M M}$ & $S I C_{L T S}$ & $S I C_{M}$ & $S I C_{L S}$ \\
\hline Case 1 & Correct fit & 50 & 72.8 & 41.1 & 17.1 & 2.0 & 100 & 85.5 & 53.2 & 15.8 & 0.5 \\
\hline & Under fit & & 4.5 & 3.7 & 31.6 & 49.2 & & 0.3 & 0.9 & 34.0 & 49.1 \\
\hline & Over fit & & 18.5 & 50.4 & 7.6 & 0.0 & & 14.1 & 45.2 & 7.1 & 0.1 \\
\hline & Wrong fit & & 4.2 & 4.8 & 43.7 & 48.8 & & 0.1 & 0.7 & 43.1 & 50.3 \\
\hline Case 2, r $=0.03$ & Correct fit & 50 & 80.7 & 44.6 & 16.7 & 4.7 & 100 & 84 & 54.3 & 15.8 & 2.7 \\
\hline & Under fit & & 0.1 & 0.2 & 32.4 & 47.7 & & 0 & 0.0 & 34.0 & 49.8 \\
\hline & Over fit & & 19.0 & 54.9 & 8.7 & 0.2 & & 16 & 45.7 & 7.2 & 0.0 \\
\hline & Wrong fit & & 0.2 & 0.3 & 42.2 & 47.4 & & 0 & 0.0 & 43.0 & 47.5 \\
\hline Case 2, r=0.1 & Correct fit & 50 & 78.5 & 42.3 & 18.2 & 5.1 & 100 & 84.5 & 53.7 & 14.6 & 3.0 \\
\hline & Under fit & & 0.1 & 0.2 & 31.4 & 44.3 & & 0.0 & 0.0 & 31.8 & 45.3 \\
\hline & Over fit & & 21.3 & 57.1 & 7.1 & 0.1 & & 15.5 & 46.3 & 8.8 & 0.0 \\
\hline & Wrong fit & & 0.1 & 0.4 & 43.3 & 50.5 & & 0.0 & 0.0 & 44.8 & 51.7 \\
\hline Case 2, r $=0.5$ & Correct fit & 50 & 80.7 & 42.6 & 9.1 & 0.7 & 100 & 81.8 & 52.6 & 5.2 & 0.0 \\
\hline & Under fit & & 0.6 & 0.7 & 16.6 & 2.8 & & 0.0 & 0.0 & 12.2 & 0.0 \\
\hline & Over fit & & 18.1 & 56.0 & 20.2 & 3.8 & & 18.2 & 47.4 & 23.9 & 3.9 \\
\hline & Wrong fit & & 0.6 & 0.7 & 54.1 & 92.7 & & 0.0 & 0.0 & 58.7 & 96.1 \\
\hline $20 \%$ bad leverage & $n$ & & $S I C_{M M}$ & $S I C_{L T S}$ & $S I C_{M}$ & $S I C_{L S}$ & $n$ & $S I C_{M M}$ & $S I C_{L T S}$ & $S I C_{M}$ & $S I C_{L S}$ \\
\hline Case 1 & Correct fit & 50 & 54.4 & 37.8 & 17.1 & 2.0 & 100 & 70.9 & 52.8 & 16.4 & 0.5 \\
\hline & Under fit & & 15.5 & 4.5 & 31.7 & 48.5 & & 8.0 & 3.4 & 32.5 & 52.4 \\
\hline & Over fit & & 9.3 & 44.0 & 6.7 & 0.1 & & 9.5 & 35.7 & 5.0 & 0.0 \\
\hline & Wrong fit & & 20.8 & 13.7 & 44.5 & 49.4 & & 11.6 & 8.1 & 46.1 & 47.1 \\
\hline Case 2, r $=0.03$ & Correct fit & 50 & 87.0 & 47.6 & 17.0 & 4.2 & 100 & 87.4 & 60.5 & 13.7 & 2.4 \\
\hline & Under fit & & 0.5 & 0.5 & 32.9 & 47.6 & & 0.0 & 0.0 & 34.3 & 47.1 \\
\hline & Over fit & & 11.8 & 50.9 & 8.5 & 0.1 & & 12.6 & 39.5 & 7.6 & 0.0 \\
\hline & Wrong fit & & 0.7 & 1.0 & 41.6 & 48.1 & & 0.0 & 0.0 & 44.4 & 50.5 \\
\hline Case 2, r $=0.1$ & Correct fit & 50 & 87.5 & 52.5 & 17.3 & 4.7 & 100 & 90.4 & 60.9 & 16.0 & 2.4 \\
\hline & Under fit & & 0.6 & 0.4 & 32.8 & 45.9 & & 0.0 & 0.0 & 31.1 & 43.6 \\
\hline & Over fit & & 11.6 & 46.7 & 7.6 & 0.2 & & 9.6 & 39.1 & 7.2 & 0.2 \\
\hline & Wrong fit & & 0.3 & 0.4 & 42.3 & 49.2 & & 0.0 & 0.0 & 45.7 & 53.8 \\
\hline Case 2, r $=0.5$ & Correct fit & 50 & 87.9 & 50.0 & 7.9 & 0.4 & 100 & 89.2 & 59.2 & 5.9 & 0.2 \\
\hline & Under fit & & 0.2 & 0.4 & 17.9 & 2.9 & & 0.0 & 0.0 & 11.5 & 0.0 \\
\hline & Over fit & & 11.3 & 48.2 & 20.2 & 3.0 & & 10.8 & 40.8 & 20.8 & 3.3 \\
\hline & Wrong fit & & 0.6 & 1.4 & 54.0 & 93.7 & & 0.0 & 0.0 & 61.8 & 96.5 \\
\hline $30 \%$ bad leverage & $n$ & & $S I C_{M M}$ & $S I C_{L T S}$ & $S I C_{M}$ & $S I C_{L S}$ & $n$ & $S I C_{M M}$ & $S I C_{L T S}$ & $S I C_{M}$ & $S I C_{L S}$ \\
\hline Case 1 & Correct fit & 50 & 43.7 & 29.6 & 17.3 & 2.6 & 100 & 44.3 & 36.7 & 16.1 & 0.7 \\
\hline & Under fit & & 20.0 & 10.9 & 30.7 & 46.9 & & 15.9 & 10.3 & 32.1 & 49.9 \\
\hline & Over fit & & 31.0 & 34.2 & 7.5 & 0.1 & & 36.5 & 26.8 & 7.9 & 0.0 \\
\hline & Wrong fit & & 5.3 & 25.3 & 44.5 & 50.4 & & 3.3 & 26.2 & 43.9 & 49.4 \\
\hline Case 2, r=0.03 & Correct fit & 50 & 75.7 & 53.3 & 18.4 & 4.5 & 100 & 91.4 & 69.6 & 17.5 & 2.3 \\
\hline & Under fit & & 6.6 & 1.5 & 29.2 & 46.6 & & 0.9 & 0.2 & 31.2 & 50.2 \\
\hline & Over fit & & 7.9 & 40.7 & 7.9 & 0.1 & & 5.7 & 29.5 & 7.9 & 0.0 \\
\hline & Wrong fit & & 9.8 & 4.5 & 44.5 & 48.8 & & 2.0 & 0.7 & 43.4 & 47.5 \\
\hline Case 2, r $=0.1$ & Correct fit & 50 & 79.2 & 54.7 & 15.9 & 3.0 & 100 & 92.8 & 70.0 & 17.6 & 1.9 \\
\hline & Under fit & & 6.2 & 1.8 & 29.3 & 44.4 & & 1.1 & 0.3 & 32.4 & 41.9 \\
\hline & Over fit & & 7.3 & 39.3 & 8.6 & 0.2 & & 4.6 & 29.0 & 7.6 & 0.0 \\
\hline & Wrong fit & & 7.3 & 4.2 & 46.2 & 52.4 & & 1.5 & 0.7 & 42.4 & 56.2 \\
\hline Case 2, r $=0.5$ & Correct fit & 50 & 83.6 & 56.1 & 8.4 & 0.4 & 100 & 94.0 & 69.1 & 5.4 & 0.0 \\
\hline & Under fit & & 1.1 & 0.6 & 16.3 & 2.6 & & 0.1 & 0.2 & 12.2 & 0.2 \\
\hline & Over fit & & 8.1 & 39.9 & 17.4 & 3.1 & & 5.1 & 30.6 & 20.5 & 3.3 \\
\hline & Wrong fit & & 7.2 & 3.4 & 57.9 & 93.9 & & 0.8 & 0.1 & 61.9 & 96.5 \\
\hline $40 \%$ bad leverage & $n$ & & $S I C_{M M}$ & $S I C_{L T S}$ & $S I C_{M}$ & $S I C_{L S}$ & $n$ & $S I C_{M M}$ & $S I C_{L T S}$ & $S I C_{M}$ & $S I C_{L S}$ \\
\hline Case 1 & Correct fit & 50 & 46.3 & 19.5 & 15.1 & 3.3 & 100 & 47.1 & 22.0 & 17.3 & 1.4 \\
\hline & Under fit & & 12.5 & 14.3 & 29.6 & 48.6 & & 11.4 & 15.2 & 30.9 & 51.2 \\
\hline & Over fit & & 36.7 & 33.1 & 11.7 & 0.0 & & 39.1 & 24.4 & 7.7 & 0.0 \\
\hline & Wrong fit & & 4.5 & 33.1 & 43.6 & 48.1 & & 2.4 & 38.4 & 44.1 & 47.4 \\
\hline Case 2, r $=0.03$ & Correct fit & 50 & 44.7 & 42.1 & 15.5 & 4.3 & 100 & 62.9 & 17.4 & 16.0 & 1.9 \\
\hline & Under fit & & 33.6 & 7.8 & 29.4 & 48.3 & & 5.0 & 34.7 & 32.8 & 46.1 \\
\hline & Over fit & & 4.9 & 31.2 & 8.9 & 0.1 & & 21.5 & 3.8 & 6.7 & 0.0 \\
\hline & Wrong fit & & 16.8 & 18.9 & 46.2 & 47.3 & & 10.6 & 44.1 & 44.5 & 52.0 \\
\hline Case 2, r $=0.1$ & Correct fit & 50 & 45.4 & 42.2 & 16.8 & 4.4 & 100 & 65.9 & 22.4 & 15.5 & 3.0 \\
\hline & Under fit & & 18.8 & 8.3 & 25.5 & 42.6 & & 4.5 & 30.6 & 30.5 & 41.6 \\
\hline & Over fit & & 31.1 & 32.1 & 9.6 & 0.1 & & 18.7 & 2.7 & 7.9 & 0.0 \\
\hline & Wrong fit & & 4.7 & 17.4 & 48.1 & 52.9 & & 10.9 & 44.3 & 46.1 & 55.4 \\
\hline Case 2, r $=0.5$ & Correct fit & 50 & 43.0 & 15.1 & 7.2 & 0.1 & 100 & 65.8 & 19.7 & 5.5 & 0.0 \\
\hline & Under fit & & 1.9 & 9.4 & 16.0 & 2.1 & & 0.9 & 3.8 & 11.6 & 0.1 \\
\hline & Over fit & & 37.0 & 11.7 & 18.7 & 3.2 & & 22.5 & 11.4 & 20.4 & 2.6 \\
\hline & Wrong fit & & 18.1 & 63.8 & 58.1 & 94.6 & & 10.8 & 65.1 & 62.5 & 97.3 \\
\hline
\end{tabular}


Mathematical Statistician and Engineering Applications

ISSN: 2326-9865

https://doi.org/10.17762/msea.v71i1.5

Table 6: Hawkins-Bradu-Kass Data

\begin{tabular}{|c|c|c|c|c|}
\hline Obs. No. & Hawkins & Bradu & kass & $y$ \\
\hline 1 & 10.1 & 19.6 & 28.3 & 9.7 \\
\hline 2 & 9.5 & 20.5 & 28.9 & 10.1 \\
\hline 3 & 10.7 & 20.2 & 31.0 & 10.3 \\
\hline 4 & 9.9 & 21.5 & 31.7 & 9.5 \\
\hline 5 & 10.3 & 21.1 & 31.1 & 10.0 \\
\hline 6 & 10.8 & 20.4 & 29.2 & 10.0 \\
\hline 7 & 10.5 & 20.9 & 29.1 & 10.8 \\
\hline 8 & 9.9 & 19.6 & 28.8 & 10.3 \\
\hline 9 & 9.7 & 20.7 & 31.0 & 9.6 \\
\hline 10 & 9.3 & 19.7 & 30.3 & 9.9 \\
\hline 11 & 11.0 & 24.0 & 35.0 & -0.2 \\
\hline 12 & 12.0 & 23.0 & 37.0 & -0.4 \\
\hline 13 & 12.0 & 26.0 & 34.0 & 0.7 \\
\hline 14 & 11.0 & 34.0 & 34.0 & 0.1 \\
\hline 15 & 3.4 & 2.9 & 2.1 & -0.4 \\
\hline 16 & 3.1 & 2.2 & 0.3 & 0.6 \\
\hline 17 & 0.0 & 1.6 & 0.2 & -0.2 \\
\hline 18 & 2.3 & 1.6 & 2.0 & 0.0 \\
\hline 19 & 0.8 & 2.9 & 1.6 & 0.1 \\
\hline 20 & 3.1 & 3.4 & 2.2 & 0.4 \\
\hline 21 & 2.6 & 2.2 & 1.9 & 0.9 \\
\hline 22 & 0.4 & 3.2 & 1.9 & 0.3 \\
\hline 23 & 2.0 & 2.3 & 0.8 & -0.8 \\
\hline 24 & 1.3 & 2.3 & 0.5 & 0.7 \\
\hline 25 & 1.0 & 0.0 & 0.4 & -0.3 \\
\hline 26 & 0.9 & 3.3 & 2.5 & -0.8 \\
\hline 27 & 3.3 & 2.5 & 2.9 & -0.7 \\
\hline 28 & 1.8 & 0.8 & 2.0 & 0.3 \\
\hline 29 & 1.2 & 0.9 & 0.8 & 0.3 \\
\hline 30 & 1.2 & 0.7 & 3.4 & -0.3 \\
\hline 31 & 3.1 & 1.4 & 1.0 & 0.0 \\
\hline 32 & 0.5 & 2.4 & 0.3 & -0.4 \\
\hline 33 & 1.5 & 3.1 & 1.5 & -0.6 \\
\hline 34 & 0.4 & 0.0 & 0.7 & -0.7 \\
\hline 35 & 3.1 & 2.4 & 3.0 & 0.3 \\
\hline 36 & 0.1 & 2.2 & 2.7 & -1.0 \\
\hline 37 & 0.1 & 3.0 & 2.6 & -0.6 \\
\hline 38 & 1.5 & 1.2 & 0.2 & 0.9 \\
\hline 39 & 2.1 & 0.0 & 1.2 & -0.7 \\
\hline 40 & 0.5 & 2.0 & 1.2 & -0.5 \\
\hline 41 & 3.4 & 1.6 & 2.9 & -0.1 \\
\hline 42 & 0.3 & 1.0 & 2.7 & -0.7 \\
\hline 43 & 0.1 & 3.3 & 0.9 & 0.6 \\
\hline 44 & 1.8 & 0.5 & 3.2 & -0.7 \\
\hline 45 & 1.9 & 0.1 & 0.6 & -0.5 \\
\hline 46 & 1.8 & 0.5 & 3.0 & -0.4 \\
\hline 47 & 3.0 & 0.1 & 0.8 & -0.9 \\
\hline 48 & 3.1 & 1.6 & 3.0 & 0.1 \\
\hline 49 & 3.1 & 2.5 & 1.9 & 0.9 \\
\hline 50 & 2.1 & 2.8 & 2.9 & -0.4 \\
\hline 51 & 2.3 & 1.5 & 0.4 & 0.7 \\
\hline 52 & 3.3 & 0.6 & 1.2 & -0.5 \\
\hline 53 & 0.3 & 0.4 & 3.3 & 0.7 \\
\hline 54 & 1.1 & 3.0 & 0.3 & 0.7 \\
\hline 55 & 0.5 & 2.4 & 0.9 & 0.0 \\
\hline 56 & 1.8 & 3.2 & 0.9 & 0.1 \\
\hline 57 & 1.8 & 0.7 & 0.7 & 0.7 \\
\hline 58 & 2.4 & 3.4 & 1.5 & -0.1 \\
\hline 59 & 1.6 & 2.1 & 3.0 & -0.3 \\
\hline 60 & 0.3 & 1.5 & 3.3 & -0.9 \\
\hline 61 & 0.4 & 3.4 & 3.0 & -0.3 \\
\hline 62 & 0.9 & 0.1 & 0.3 & 0.6 \\
\hline 63 & 1.1 & 2.7 & 0.2 & -0.3 \\
\hline 64 & 2.8 & 3.0 & 2.9 & -0.5 \\
\hline 65 & 2.0 & 0.7 & 2.7 & 0.6 \\
\hline 66 & 0.2 & 1.8 & 0.8 & -0.9 \\
\hline 67 & 1.6 & 2.0 & 1.2 & -0.7 \\
\hline 68 & 0.1 & 0.0 & 1.1 & 0.6 \\
\hline 69 & 2.0 & 0.6 & 0.3 & 0.2 \\
\hline 70 & 1.0 & 2.2 & 2.9 & 0.7 \\
\hline 71 & 2.2 & 2.5 & 2.3 & 0.2 \\
\hline 72 & 0.6 & 2.0 & 1.5 & -0.2 \\
\hline 73 & 0.3 & 1.7 & 2.2 & 0.4 \\
\hline 74 & 0.0 & 2.2 & 1.6 & -0.9 \\
\hline 75 & 0.3 & 0.4 & 2.6 & 0.2 \\
\hline
\end{tabular}

Vol. 71 No. 1 (2022) 
Table 7: The values of different criteria for Hawkins-Bradu-Kass data for different set of variables

\begin{tabular}{c|cccc}
\hline Set of variables & $S I C_{M M}$, & $S I C_{L T S}$ & $S I C_{M}$ & $S I C_{L S}$ \\
\hline$(y$, Hawkins $)$ & -0.3877 & -0.6233 & 0.8786 & 1.7553 \\
$(y$, Bradu $)$ & -0.4056 & -0.6277 & 0.2157 & 1.8609 \\
$(y$, Kass $)$ & -0.3982 & -0.5835 & -0.3089 & 1.7077 \\
$(y$, Hawkins,Bradu $)$ & -0.3525 & -0.5904 & -0.2360 & 1.7898 \\
$(y$, Hawkins,Kass $)$ & -0.3999 & -0.6514 & $\mathbf{- 0 . 1 1 4 7}$ & 1.7358 \\
$(y$, Bradu,Kass $)$ & -0.3766 & $\mathbf{- 0 . 5 7 1 9}$ & -0.2206 & $\mathbf{1 . 6 8 3 9}$ \\
$(y$, Hawkins,Bradu,Kass $)$ & $\mathbf{- 0 . 4 0 6 2}$ & -0.6816 & -0.1385 & 1.7077 \\
\hline \hline & & & &
\end{tabular}

